\title{
Mapas conceptuales y habilidades de pensamiento en la formación continua de los docentes en un programa de posgrado
}

\section{Concept maps and thinking skills in continuing teacher education in a graduate program}

\author{
DOI: $10.46932 / s f j d v 3 n 2-005$
}

Received in: February $28^{\text {th }}, 2022$

Accepted in: March $1^{\text {st }}, 2022$

\section{Rodolfo Huerta González \\ Maestría en Historia}

Institución: UPN, Unidad 151-Toluca Sede Regional Acambay

Dirección: Mariano Salgado s/n Libertad, Deleg. Santa Cruz Atzcapotzaltongo, CP. 50030, Municipio de Toluca de Lerdo, Estado de México

Correo electrónico: rhuerta12@gmail.com

\section{María Guadalupe Mendoza Ramírez \\ Doctorado en Ciencias Sociales \\ Institución: UPN, Unidad 151-Toluca}

Dirección: Mariano Salgado s/n Libertad, Deleg. Santa Cruz Atzcapotzaltongo, CP. 50030, Municipio de Toluca de Lerdo, Estado de México

Correo electrónico: mmendozaupntoluca@gmail.com

\section{RESUMEN}

El presente artículo analiza la experiencia desarrollada con los maestrantes de la Maestría en Educación Básica en la Universidad Pedagógica Nacional Unidad 151-Toluca, que cursaron del primero al cuarto cuatrimestre en 2020-21, con el objetivo de mejorar la elaboración de mapas conceptuales. El diseño de investigación abordó la elaboración de mapas conceptuales al inicio del programa de Maestría; en el transcurso se les pidió la elaboración de otros dos mapas. Se tomó un estudio de caso con el profesor que presentó serias dificultades en los dos primeros mapas. Los resultados muestran que el uso de habilidades de pensamiento ayudó sustancialmente a mejorar la elaboración de los mapas conceptuales del profesor.

Palabras clave: mapas conceptuales, aprendizaje significativo, habilidades de pensamiento.

\begin{abstract}
This article analyzes the experience developed with the teachers of the Master's Degree in Basic Education at the Universidad Pedagógica Nacional Unidad 151-Toluca, who attended from the first to the fourth semester in 2020-21, with the aim of improving the elaboration of concept maps. The research design addressed the development of concept maps at the beginning of the Master's program; in the course they were asked to prepare two other maps. A case study was taken with the teacher who presented serious difficulties in the first two maps. The results show that the use of thinking skills substantially helped to improve the preparation of the teacher's concept maps.
\end{abstract}

Keywords: concept maps, meaningful learning, thinking skills. 


\section{INTRODUCCIÓN}

La Maestría en Educación Básica (MEB) que se oferta en la UPN, Unidad 151-Toluca tiene un carácter profesionalizante, es decir, que debe reunir los rasgos de: atención de necesidades de educación y el desempeño de los egresados en una actividad laboral afín a su formación (Pérez, 2018, p. 111). En esta manera, en el primer año los profesores realizan un análisis de su propia práctica docente y, al mismo tiempo, adquieren un conjunto de herramientas teórico-metodológicas para que reflexionen, transformen y mejoren su labor docente a través del diseño e implementación de una propuesta innovadora.

El documento rector de la MEB establece que el profesor: "profundice en la comprensión de los procesos educativos y desarrolle la capacidad reflexiva para transformar su práctica profesional, renovar y construir conocimientos, desarrollar actitudes, valores y habilidades que integren los saberes propios de su práctica, en el marco de una formación por competencias" (UPN, 2012, p. 4). De esta forma, la MEB fomenta la búsqueda de áreas de oportunidad para la actualización y profundización en teorías, metodologías, saberes educativos $\mathrm{y}$, en este caso, el desarrollo de competencias en programas comprendidos en las Tecnologías del Aprendizaje y el Conocimiento (TAC's).

Las TAC's implican el uso de las tecnologías de la información y la comunicación (TIC's) hacia procesos más formativos, como para el estudiante y el profesor, con el fin de aprender más y mejor, con el dominio de una serie de herramientas informáticas. Es decir, explorar, analizar y evaluar los posibles usos didácticos que estas herramientas ofrecen al aprendizaje y la docencia (Lozano, 2011, p. 45).

Una de las debilidades detectadas por los propios profesores fue la elaboración de mapas conceptuales, por lo que se implementó en tres primeros trimestres (de seis) del primer y segundo año de la maestría varias sesiones para construir de manera personal y colaborativa mapas conceptuales según las especificaciones de Novak, Cañas y colaboradores (2005, 2006, 2008) usando el software libre CmapTools y aplicando seis habilidades de pensamiento: elaboración de preguntas de enfoque, análisis, abstracción, jerarquización, conceptualización y relación.

\section{PROBLEMATIZACIÓN}

Cuando inician la maestría, se les pide a los maestrantes la realización de un mapa conceptual para valorar su conocimiento previo de este tema. La casi totalidad de los mapas que entregan, carecen de una pregunta enfoque, los elaboran como si fueran organigramas, consideran como conceptos verbos o frases, no establecen variados niveles de jerarquía, no ponen conectores entre los conceptos y cuando los anotan solo son conjunciones o artículos, y generalmente desarrollan tres líneas de análisis. Cuando se les pregunta el por qué los hicieron de esta forma, señalan que así se los enseñaron en su formación inicial o 
que depende de qué autor leyeron, pero al preguntarles qué autores han consultado, no pueden nombrar alguno.

Lo anterior sugiere que la incorporación de mapas conceptuales por parte de los profesores, impulsada desde la reforma educativa de 1992-93 (SEP, pp. ), para la enseñanza de diversas asignaturas en la Educación Básica, no muestra avances significativos en los niveles de aprendizaje de los docentes de la teoría educativa subyacente, como tampoco se ven en la apropiación metodológica que hacen para enseñarlos de una forma didácticamente adecuada a sus alumnos, lo que conlleva a cuestionar si los profesores y sus alumnos aprenden significativamente.

Por otro lado, los manuales divulgadores que puede ser consultados para la elaboración de mapas conceptuales tiene sus propios errores; por ejemplo, Hernández (2007, p. 62) presenta los mapas conceptuales como mapas mentales con el concepto más inclusivo en el centro del mapa, y de forma irradiante los conceptos secundarios, incluyendo un buen número de figuras. En este mismo sentido, Gutiérrez (2001) muestra los mapas conceptuales con la estructura de un organigrama y, además, propone a los alumnos de preparatoria que no usen palabras de enlace (p. 80); sugiere, además, nombres propios como conceptos: "Lázaro Cárdenas", "Plutarco Elías Calles”; el uso de años como conceptos “1920”, “1925”, “1936”; y también oraciones: "se crea la Liga Nacional de la Defensa religiosa”, “aprobó un enfoque marxista a la enseñanza de la historia" (p. 101). La forma de organigrama como mapa conceptual que proponen los autores es la siguiente:

Gráfico 1. Modelo de organigrama usado habitualmente como mapa conceptual.

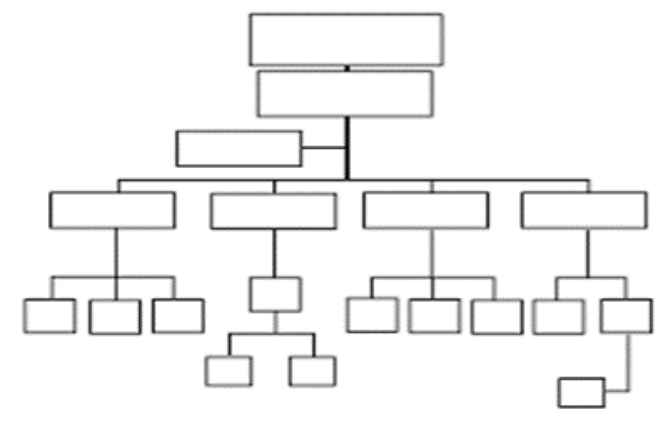

Asimismo, Boggino (2006) considera, en varios ejemplos, como conceptos algunas oraciones: "representar las relaciones significativas", (p. 22); "unir lo diverso" (p. 88); usa un verbo como el concepto más inclusivo: “Globalizar” (p. 88), y sus jerarquías corresponden a un organigrama. En otro caso, Maya y Díaz (2013) usan nombres personales: “Julio César” (p. 78); “Grecia”, "Pompeya”, “el Vesubio”, “Hércules" (p. 79). Además, en estas cuatro obras citadas no se propone la redacción de una pregunta de enfoque que guie la construcción del mapa. Todos ellos difieren sustancial de la propuesta original de Novak y sus colaboradores. 


\section{MARCO TEÓRICO}

El concepto de aprendizaje significativo tuvo dos formulaciones. En las primeras obras de Ausubel (1968, 1976 en español), lo definió como: “... si la tarea de aprendizaje puede relacionarse, de modo no arbitrario, sustantivo (no al pie de la letra), con lo que el alumno ya sabe...” (pp. 40-41). La parte que decía: "con lo que ya sabe", era muy general porque el alumno puede saber muchas cosas, pero no identificar cuál de esas puede servirle para aprender significativamente.

Por eso, en una de sus últimas obras publicadas, Ausubel (2010, 2002 en español) lo redefinió como: “... las ideas potencialmente significativas del material de instrucción se relacionan selectivamente con ideas pertinentes y también más generales e inclusivas ya existentes (denominadas éstas por Ausubel como ideas de anclaje) en la estructura cognitiva" (p. 35). Es decir, que de todas las ideas que han creado, los alumnos deben seleccionar las más pertinentes (que le van a servir de anclaje) para relacionarlas con las ideas significativas del material de instrucción.

Ausubel reconoce que hay tres tipos de aprendizaje significativo: el de las representaciones, de los conceptos y el de las proposiciones. El de representaciones ocurre cuando los símbolos arbitrarios (letras, números, señales, etc.) se igualan en su significado con sus referentes (personas, objetos, eventos, conceptos), es decir, que todo tiene un nombre que significa lo que su referente implica para una persona en particular (Ausubel, Novak y Hanesian, 1983, p. 46).

Los conceptos instituyen un elemento de mayor importancia en la teoría de la asimilación porque la comprensión y la resolución significativa de problemas estriban en gran medida de la disponibilidad, en la estructura cognitiva del estudiante, de conceptos de orden superior (en la adquisición subsumidora de conceptos) y de conceptos subordinados (en la adquisición de conceptos de orden superior). En un sentido muy parecido, Novak propone que los elementos básicos del conocimiento son los conceptos, y las relaciones entre conceptos llamadas proposiciones (Cañas, 2005, p 2).

Por lo anterior, es indudable: 1) que los seres humanos dilucidan las experiencias perceptivas en función de unos conceptos particulares de sus estructuras cognitivas (coincidiendo con Piaget), y 2) que los conceptos son los componentes básicos tanto del aprendizaje significativo basado en la recepción de propiedades declarativas como de la generación de proposiciones significativas en la resolución de problemas (Ausubel, 2002, p. 27).

Y, por último, para Ausubel (2002) el más importante de los aprendizajes significativos es el proposicional que, al igual que el conceptual, puede ser subordinado, supraordinado o combinatorio. El aprendizaje subordinado se promueve cuando una proposición lógicamente significativa de una disciplina particular se relaciona significativamente con unas proposiciones específicas de orden superior (o supraodinadas) en la estructura cognitiva del estudiante. 
El aprendizaje proposicional supraordinado se logra cuando una proposición nueva se puede vincular o bien con unas ideas subordinadas determinadas de la estructura cognitiva ya existente o bien con un desarrollado conjunto de ideas pertinentes de la estructura cognitiva que se pueden subsumir en ella. Por último, el aprendizaje proposicional combinatorio se refiere a los temas en los que una proposición potencialmente significativa no es enlazable con unas ideas específicas subordinadas o supraordenadas en la estructura cognitiva del estudiante, pero sí lo es con una combinación de contenidos pertinentes en general, pero también menos pertinentes, de esa estructura (Ausubel, 2002, p. 28).

Los mapas conceptuales desarrollan los dos últimos tipos de aprendizaje. Y aunque Ausubel (2002) afirme que indudablemente, la mayor parte del aprendizaje proposicional es subsumidor o combinatorio (p. 28), su teoría es bastante atractiva si se piensa que si toda la información que se almacena en la mente humana en mapas conceptuales donde hay ya conceptos y proposiciones sub y supraordinadas, es precisamente esta organización jerárquica la que nos permitirá incorporar nuevas informaciones en función de la ampliación constante de la retícula de mapas conceptuales lo que se volvería una forma de aprender más y mejor cada día.

La otra faceta teórica son las habilidades de pensamiento (HP). Las HP pueden definirse como el conjunto de acciones interiorizadas, organizadas y reguladas, que favorecen un adecuado procesamiento de la información, administradas tanto a la información a procesar en sí, como a las estructuras, procesos y estrategias que están siendo usadas al procesarla (Lipman, 1991, p. 153).

Así, las habilidades de pensamiento se refieren tanto a las acciones para el procesamiento de la información como a los procesos y estrategias cognitivos que usa la mente humana para ese procesamiento. De esta manera, no solo son exclusivas para el procesamiento y uso de la información sino fundamentalmente para la creación de nuevo conocimiento.

Un análisis de diversos mapas conceptuales construidos por docentes en servicio en educación primaria y profesores-estudiantes en esta maestría, han posibilitado la idea de que es posible desarrollar al menos 11 habilidades de pensamiento con los mapas conceptuales: elaboración de preguntas de enfoque, análisis, abstracción, jerarquización, conceptualización, relación, comparación, abstracción, clasificación, deducción, inducción y síntesis. Sin embargo, las HP que fueron tácitamente desarrolladas en este trabajo fueron las seis primeras.

Por otro lado, el núcleo central de los mapas conceptuales está constituido por los conceptos y la formulación de proposiciones entre estos. El concepto es definido como una regularidad en los acontecimientos o en los objetos, que se designa mediante algún termino (Novak y Gowin, 1998, p. 26). La idea de Ausubel es que los mapas conceptuales representan relaciones significativas entre conceptos en forma de proposiciones. Una proposición consta de dos o más términos conceptuales unidos por 
palabras para formar una unidad semántica, por lo que el mapa es un esquema que representa un conjunto de significados conceptuales incluidos en una estructura de proposiciones (Novak y Gowin, 1998, p. 33).

Así, son herramientas gráficas para organizar y representar el conocimiento. Incluyen conceptos, encerrados en círculos o elipses y las relaciones entre los conceptos indicados por una línea que conecta dos o más conceptos; las palabras sobre la línea orientan la vinculación dada por las palabras, frases u oraciones de enlace, y que fijan la relación entre los dos conceptos, creando una proposición. Las proposiciones son afirmaciones que se hacen sobre algún objeto, evento o proceso, ya sea natural o construido. Las proposiciones así construidas constituyen una declaración significativa por lo que se denominan unidades semánticas o unidades de significado (Novak y Cañas, 2008, p. 1).

Los principios de aprendizaje claves de la psicología cognitiva de Ausubel que están presentes en los mapas son:

- El aprendizaje significativo (a diferencia del aprendizaje mecánico) es ineludible para el desarrollo de un entendimiento conceptual. El aprendizaje significativo es acreditado a veces como aprendizaje profundo o dinámico (en oposición con el aprendizaje superficial o estático).

- El nuevo aprendizaje se debe cimentar sobre conceptos y proposiciones previas relevantes construidas por el aprendiz.

- $\mathrm{Al}$ aprendiz se le debe incentivar para que opte por aprender significativamente.

- Se requieren ayudas oportunas para aprender conceptos abstractos, adyacente con la instrucción didáctica apropiada.

- El aprendizaje es altamente idiosincrático y prospera con el tiempo.

- El aprendizaje significativo de alta calidad traslada a la construcción de estructuras conceptuales y proposicionales bien integradas (es decir, estructuras cognitivas) que proporcionan mejor el nuevo aprendizaje y la solución creativa de problemas (Cañas y Novak, 2006, s. p.)

La característica fundamental de los mapas conceptuales es que están construidos de una forma jerárquica, los conceptos más inclusivos (los que incluyen a los otros) van en la parte superior del mapa y hacia abajo, los conceptos menos inclusivos, más específicos, organizados jerárquicamente. La mejor forma de elaborar mapas conceptuales es partir de una pregunta particular que se busca responder, llamada pregunta de enfoque. En este sentido, el mapa conceptual debe pertenecer a alguna situación o evento que estamos tratando de conocer o de aprender a través de la organización jerarquizada del conocimiento a través de un mapa conceptual.

Otra característica importante de los mapas conceptuales es la inclusión de enlaces cruzados. Estas son relaciones o vínculos entre los conceptos en diferentes ramas de conceptos. Los enlaces cruzados nos ayudan a ver como un concepto de una rama de conocimiento representada en el mapa está relacionado 
con un concepto en otro dominio mostrado en el mapa. Los enlaces cruzados, en la creación de nuevos conocimientos, representan saltos creativos por parte del elaborador del mapa. De hecho, hay dos características de los mapas conceptuales que son importantes en la facilitación del pensamiento creativo: la estructura jerárquica que está representada por un buen mapa y la capacidad para buscar y caracterizar nuevos enlaces cruzados.

Una última característica que puede ser añadida a los mapas conceptuales son los ejemplos específicos de eventos, objetos o situaciones que ayudan a aclarar el significado de un concepto dado. Normalmente, estos no están incluidos en óvalos o rectángulos con puntas redondeadas, ya que son eventos u objetos específicos y no representan conceptos (Novak y Cañas, 2008, p. 2).

Los mapas conceptuales son eficaces también en la identificación de ideas válidas y no válidas en poder de los estudiantes (Novak y Cañas, 2008, p. 6). También han sido usados para determinar los conocimientos previos de los estudiantes en diversas disciplinas y asignaturas, como formas de evaluación de la asimilación de conocimientos, para captar el significado de los materiales que se van a aprender, como instrumentos poderosos para la comprensión de textos y para desarrollar habilidades de pensamiento crítico en estudiantes de una lengua extranjera (Novak y Gowin, 1998, p. 19; Novak y Cañas, 2008, p. 15; Cañas, 2005, p. 4; Khodafy \& Ghanizafeh, 2011, p. 49).

\section{PROCEDIMIENTO}

La experiencia de formación en mapas conceptuales que se analiza en este artículo se llevó a cabo con el grupo de maestría de la primera especialización de la MEB. Se les pidió que elaboraran un mapa tal como los conocían de uno de los temas que se estaban analizando en ese módulo, que sirvió para determinar los conocimientos previos en la elaboración de mapas conceptuales. La única indicación fue que lo elaboraran en CmapTools, un software desarrollado por Novak, Cañas y sus colaboradores en el Instituto de la Cognición Humana y de la Máquina.

Se muestra un mapa conceptual inicial de un maestrante, egresado de licenciatura de la UPN. 
Figura 1 Mapa conceptual inicial

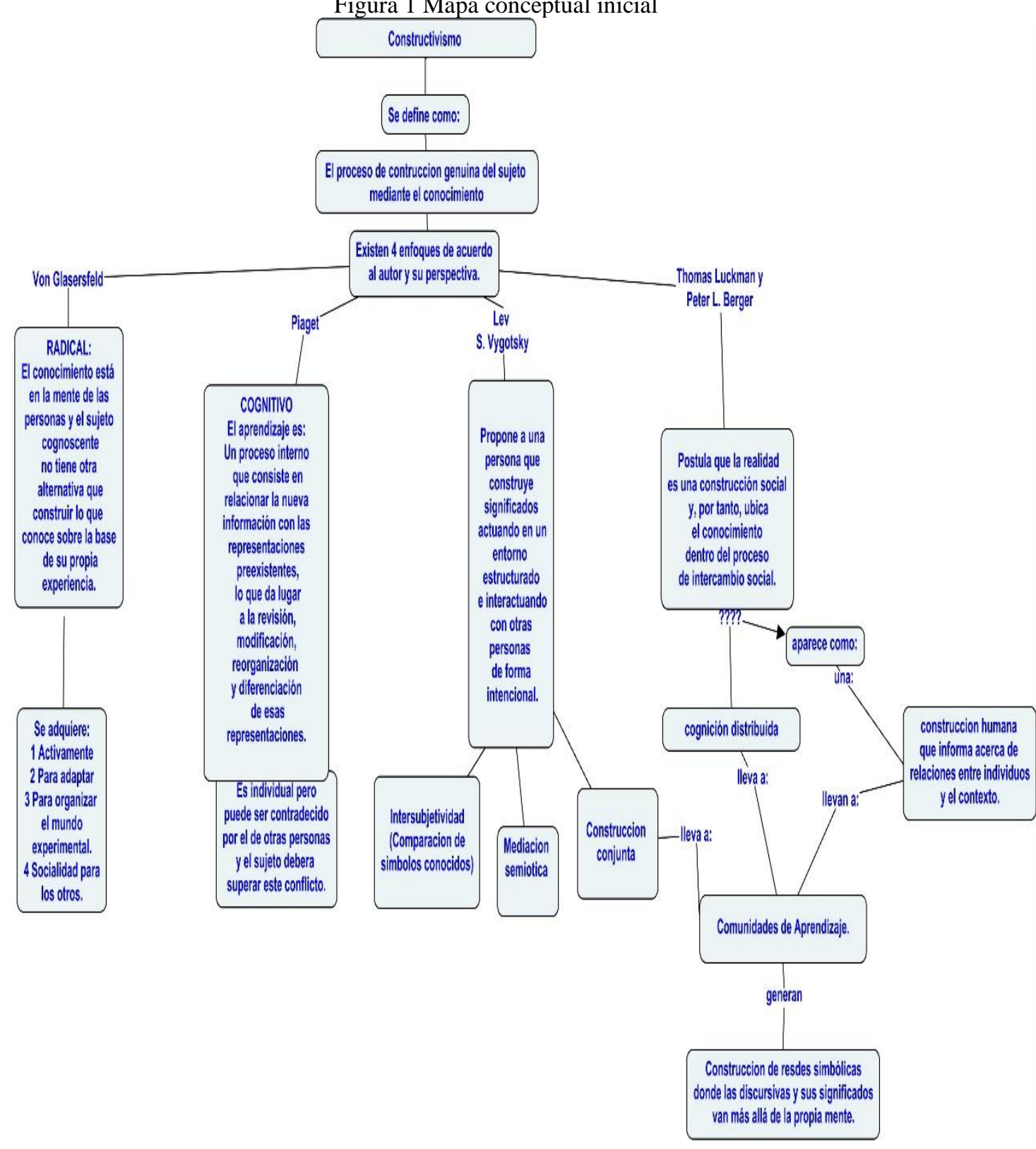

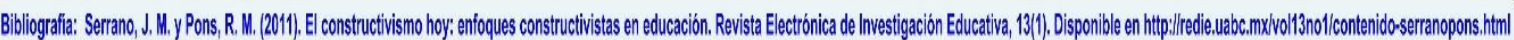

Al analizar el mapa inicial puede observarse que no inicia con una pregunta de enfoque. El concepto más inclusivo es correcto, pero luego sigue una oración "se define como:"; las siguientes elipses no son conceptos sino son una frase "El proceso de construcción genuina del sujeto mediante el conocimiento", y otra oración "Existen 4 enfoques de acuerdo al autor y perspectiva". En la frase que escribe están incluidos tres conceptos, pero no los toma en consideración: "proceso de construcción", "sujeto" y "conocimiento". No cuentan con conectores estas cuatro elipses, porque los artículos y verbos 
están incorporados como como si fueran conceptos. Es visible también la dificultad para conceptualizar, relacionar y crear proposiciones.

Despliega cuatro líneas de análisis según los autores mencionados en la lectura: Von Glasersfeld, Piaget, Lev. S. Vigotsky, y Thomas Luckman y Peter L. Berger, retomando la estructura del artículo ya fijada por los escritores del mismo. Pero en los siguientes cuatro rectángulos redondeados escribe en cada uno de ellos un breve resumen de un párrafo seleccionado copiando algunos de los postulados; por ejemplo, un concepto para el maestrante es el siguiente: "El conocimiento está en las mentes de las personas y el sujeto cognoscente no tiene otra alternativa que construir lo que conoce sobre la base de su propia experiencia”.

En la siguiente línea de jerarquía tiene seis rectángulos redondeados, pero solo tres contienen conceptos: "Intersubjetividad (comparación de símbolos conocidos)", "mediación semiótica", "construcción conjunta" y. En la última rama, la de la derecha, tiene dos conceptos "comunidad de aprendizaje", "cognición distribuida", y tres oraciones: “aparece como", "construcción humana...", "Construcciones de resdes (sic) simbólicas", y repite como enlace tres veces "lleva a".

La aplicación de las habilidades de pensamiento en el desarrollo de este mapa conceptual es muy reducida: no hay pregunta de enfoque, solo tiene cuatro ramas de análisis pero son las establecidas por los autores del artículo publicado y como no hay mucho análisis no hay tampoco abstracción más que en las líneas 3 ("Intersubjetividad (comparación de símbolos conocidos)", "mediación semiótica", "construcción conjunta") y 4 (“cognición distribuida" y "construcción humana que informa acerca de las relaciones entre individuos y el contexto").

Por otro lado, tienen ocho niveles de jerarquía, pero porque sigue el desarrollo ofrecido por los autores; en cuanto a la conceptualización, de 18 colocados como conceptos solo seis lo son, lo que significa un tercio correcto en el uso de conceptos. En cuanto a la relación solo escribió nueve conectores de los cuales cuatro era nombres personales y tres fueron "lleva a".

Por lo tanto, se puede concluir que el limitado conocimiento y aplicación de habilidades de pensamiento no llevó a obtener aprendizajes significativos a pesar de ser una poderosa estrategia el mapa conceptual. Se presentan asimismo oraciones y frases en los rectángulos redondeados, es decir, se usan como conceptos cuando no lo son.

Esto suele indicar el uso de párrafos enteros con la intención de presentar un resumen (actitud que sugiere una tendencia a copiar las líneas completas y pegarlas tal cual, sin reelaborar o reestructurar la información) en lugar de proposiciones entre conceptos. Lo anterior sugiere serias dificultades para conceptualizar. Indican también falta de análisis y síntesis en la lectura del material fuente del mapa conceptual así como una estructuración inadecuada del mismo. 
En cuanto a las ideas de Ausubel de los aprendizajes proposicionales subordinados, supraordinados y combinatorios en este mapa conceptual carecen de algún sentido, por motivo de que el mapa conceptual tiene pocos conceptos y más frases y oraciones. Como los aprendizajes proposicionales, cualquiera de ellos, se forman en la relación de dos o más conceptos y ya que casi no hay conceptos, pues no hay este tipo de aprendizaje. En las dos únicas proposiciones donde se relacionan dos conceptos (“cognición distribuida lleva a comunidades de aprendizaje" y "construcción conjunta lleva a comunidades de aprendizaje) reflejan que no hubo comprensión de la lectura y una concepción errónea de lo que son los mapas conceptuales.

Este ejemplo de mapa conceptual muestra igualmente una actitud que favorece el aprendizaje mecánico por parte del maestrante al resumir algunas ideas propias de los autores del artículo, y hacerlas pasar por conceptos sin la respectiva reflexión de lo que debe hacerse en la construcción de un mapa conceptual; el escaso intento de construir proposiciones lógicas sustituidas por el copiado y pegado de frases y oraciones que impidieron la construcción de significados propios por el maestrante y, por lo tanto, la construcción mecánica, sin sentido, de un mapa conceptual.

La explicación específica de las HP radicó en exponerles cómo desarrollarlas en la construcción de mapas conceptuales. Principalmente consistió en investigar y discutir cuáles eran las habilidades de pensamiento que pudiesen desarrollarse a través de mapas conceptuales para que en su proyecto de intervención los aplicaran y evaluaron el logro de dichas habilidades en sus alumnos. La guía de investigación consistió en determinar algunas acepciones de las 11 habilidades de pensamiento previamente identificadas en cursos anteriores las cuales pudieran ser aplicadas específicamente en la elaboración de mapas conceptuales en los niveles de Educación Básica, las cuáles son: 
Tabla. 1. Habilidades de Pensamiento y mapas conceptuales.

Habilidad de pensamiento

Elaboración de preguntas de enfoque

Análisis

Abstracción

Jerarquía

Conceptualización

\section{Procedimiento}

Es una forma de delinear el contexto (qué queremos aprender al hacer un mapa conceptual) al construir una Pregunta de Enfoque, esto es, una pregunta que especifique claramente el problema o asunto el mapa conceptual ayudará a resolver. Para el desarrollo del mapa conceptual, se puede definir la pregunta de enfoque, con las preguntas de indagación como "¿dónde?, ¿qué cosa?, ¿quién?, ¿cómo’, ¿por qué?" pues ayudan a establecer lo que el estudiante ya sabe y lo que no; también pueden ser preguntas de verificación que se usan para comprobar si las proposiciones en el mapa conceptuales son verdaderas o no y si son coherentes. Las preguntas de amplificación como ¿las proposiciones son lógicas? ¿me queda clara la información? Que ayudan a determinar si falta información, si faltan conceptos, o si deben establecerse enlaces cruzados. También la pregunta de enfoque puede elaborarse si el mapa será descriptivo, explicativo o comprensivo. Por ejemplo, la pregunta puede ser ¿Qué es la sociedad?, ¿Por qué surge la sociedad? o ¿Cómo funciona la sociedad? Otro ejemplo sería ¿Cuáles son las partes de las plantas? ¿Para qué sirven las plantas? ¿Cómo se lleva a cabo la fotosíntesis en las plantas? Las buenas preguntas estimulan altos niveles de aprendizaje dinámico y significativo.

De la lectura, el alumno determina el concepto más inclusivo de primer orden, después va creando ramas distintas en función de conceptos menos inclusivos, que forman la idea de la creación de un árbol invertido. Dependiendo de la riqueza analítica de la lectura y del lector, se distinguirán varias ramas y sus subsecuentes ramificaciones. Generalmente los alumnos no expertos determinan de tres a cinco ramas sin tomar en cuenta la calidad de la lectura. El análisis se vincula con la relación y la jerarquización.

Esta habilidad se desarrolla cuando de un concepto menos inclusivo (que es una parte de uno más inclusivo) lo consideramos nuevamente como un todo y separamos sus partes creando nuevas ramas en el mapa conceptual, en el sentido de un árbol invertido. Y este mismo proceso, lo llevamos a cabo en los siguientes niveles inferiores de inclusividad. De hecho, la abstracción está íntimamente relacionada con el análisis. Establecer los diferentes niveles de inclusividad de los conceptos, del más inclusivo, o abstracto, a los menos inclusivos o concretos. Los conceptos más inclusivos son los supraordinados y los más específicos los subordinados. El concepto más inclusivo, inclusor o supraordinado va hasta arriba y hacia abajo siguen los más específicos o subordinados. De esta manera, en los mapas conceptuales quedan concretadas las ideas del aprendizaje supraordinado y subordinado desarrolladas por Ausubel. El aprendizaje combinatorio sucede cuando el alumno hace líneas cruzadas entre diferentes ramas, donde no hay aprendizaje subordinado ni supraordinado. La jerarquía en los mapas conceptuales no es rígida como sucede en los organigramas donde los niveles jerárquicos están siempre a una equidistancia de un nivel al otro. En los mapas conceptuales la jerarquía es múltiple, no sigue un patrón prestablecido, sino que depende de la inclusividad de los conceptos mismos. La jerarquía se vincula íntimamente con el análisis y la relación.

Es determinar el uso adecuado de etiquetas que señalan regularidades de personas, objetos, situaciones, etc. Uno de los problemas para el desarrollo de esta habilidad es que los alumnos deben entender que los verbos, los nombres personales y fechas, no son conceptos; en el caso de los verbos, que son acciones (jugar, comer, pensar, ir) comúnmente confundidos como conceptos, pueden convertirse en conceptos: mover (verbo) en movimiento (concepto), pensar (verbo) en pensamiento (concepto); comer (verbo) en comida (concepto). Este es también un proceso de conceptualización. 
En otro caso, el desarrollo de la habilidad se comprueba cuando no se usan oraciones o frases como conceptos. La conceptualización implica un grado alto de abstracción.

Relación

Es la habilidad de unir adecuadamente dos conceptos y crear proposiciones lógicas desde el punto de vista comunicativo. No implica necesariamente el uso adecuado de proposiciones, artículos o conjunciones, sino la claridad conceptual del alumno. Sin embargo, la forma en que el estudiante relacione los conceptos y forme las proposiciones, le dará el significado específico que él ha construido y depende del contexto o del problema que quiera resolver y del nivel enfoque y de reflexión de quien lo está elaborando. La habilidad va estrechamente ligada con el análisis y la conceptualización pues sin ambas, el alumno construye proposiciones sin sentido comunicativo alguno. Hay que decir que la relación entre conceptos y, por consiguiente, la calidad de la proposición depende de la idiosincrasia del que hace el mapa conceptual.

Nota: Adaptado de El proceso de la investigación científica por R. Rojas, p. 79; "La teoría subyacente a los mapas conceptuales y como construir y utilizarlos" por J. Novak y J. A, Cañas, 2008, p. 2; Aprendiendo a aprender por J. D. Novak y B. Gowin, 1998, p. 22; "Re-examinando los fundamentos para el uso efectivo de mapas conceptuales" por J. A. Cañas y J. Novak, 2006, s. p.; "Mapas conceptuales para favorecer el aprendizaje en Ciencias de la Salud" por V. Luna, 2014, p. 221.

Después de dos sesiones de dos horas cada una de capacitación en las ideas de Novak y Cañas (adicionalmente tuvieron tres horas para aprender el CmapTools) para la elaboración de mapas conceptuales y un trimestre y medio analizando y aplicando en diversos temas las seis habilidades que como maestrantes tienen que hacer una intervención en sus centros laborales desarrollando habilidades de pensamiento, se les pidió nuevamente la elaboración de otro mapa conceptual. La siguiente figura muestra el nuevo mapa conceptual realizado de la misma lectura. 
Figura 2 Mapa reelaborado por el mismo maestrante

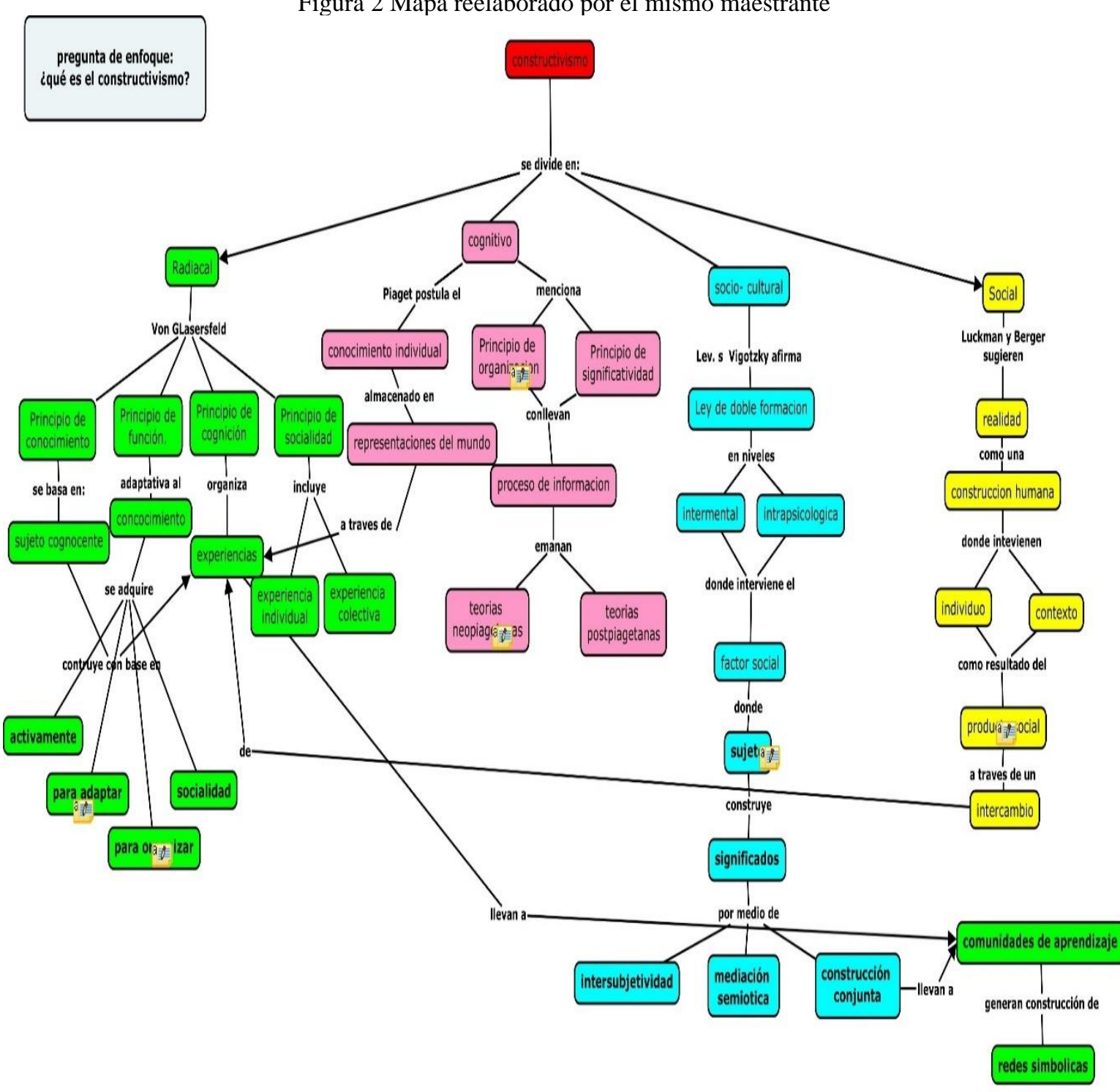

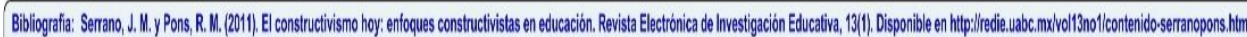

Si comparamos los dos mapas conceptuales se puede apreciar un avance notable en la aplicación de habilidades de pensamiento, aunque hay algunos retos aún que superar. De inicio, es un mapa que se acerca a la idea original de los creadores de los mapas conceptuales. Incluye ya la pregunta de enfoque, aunque no hay una coherencia plena entre la pregunta y el contenido del mapa pues más bien no se dilucida que es el constructivismo y la información habla de cuatro tipos de constructivismo con sus principales postulados.

Con respecto a la habilidad de análisis, hay por lo menos ocho ramas que implican una mayor descomposición del todo en partes, siendo más visible en los dos primeros tipos de constructivismo: radical y cognitivo, aunque disminuye sensiblemente en las dos ramas siguientes ("socio-cultural" y "Social"). Ya que hay mayor análisis que en el mapa conceptual, también ya hay abstracción que se 
muestra al ver el concepto "radical" como un todo y descomponerlo en cuatro ramas ("principio de conocimiento", "principio de función”, “principio de cognición” y “principio de socialidad”) y más abajo del concepto conocimiento salen cuatro, aunque los tres primeros no lo son: "activamente", "para adaptar", "para organizar" y "socialidad”); en la línea del "cognitivo" se descompone en "conocimiento individual”, "principio de organización” y “principio de significatividad".

Con respecto a la habilidad de jerarquización, hay avances importantes pues muestra 25 niveles de jerarquía, aunque un análisis más fino mostraría que son unos 18, porque el maestrante aún no dominó adecuadamente el software ya que se aprecia ciertas dificultades aún para emparejar los conceptos de la misma jerarquía, como se aprecia en los conceptos de

“activamente” (que no es concepto), "para adaptar" (que no es concepto), "para organizar” (que no es concepto), "socialidad", que no pudo ponerlos en el mismo nivel de jerarquía.

Tabla 2 Evaluación de las habilidades de pensamiento.

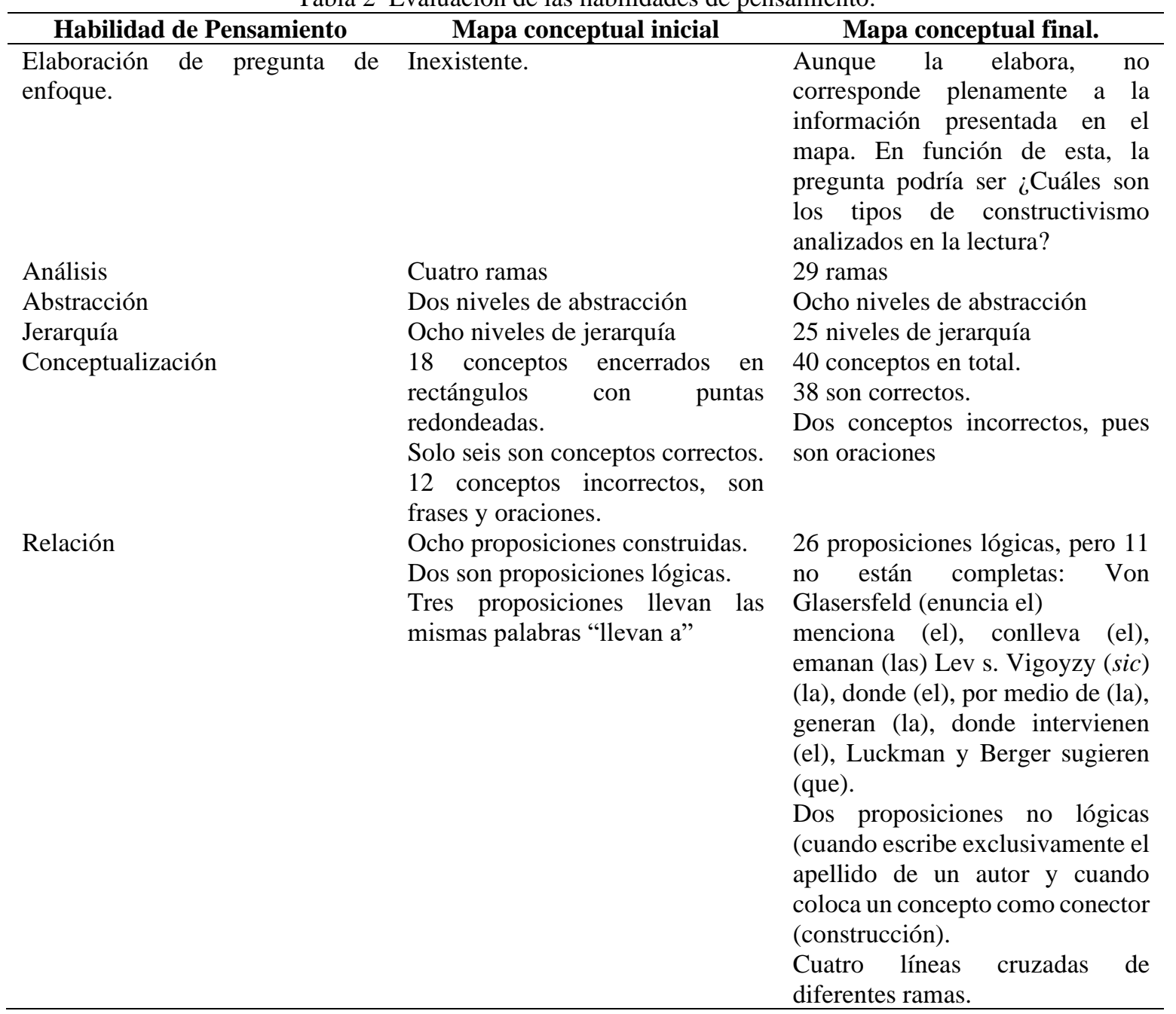

Es importante señalar que el esfuerzo de la mediación pedagógica ofrecida en los cursos de la MEB en relación al uso de las habilidades del pensamiento, principalmente las de abstracción, 
jerarquización y relación, para la elaboración de mapas conceptuales influyó en la construcción fundamentalmente de conceptos y proposiciones subordinadas y de proposiciones combinatorias. En ambos casos, la diferencia entre el mapa inicial y el posterior es muy marcada.

Por ejemplo, la presencia de proposiciones subordinadas en el primero era inexistente como pasaba igual con la pregunta de enfoque. Las proposiciones eran inexistentes porque no había conectores entre los rectángulos redondeados porque las palabras de enlace estaban incluidas como conceptos formando frases u oraciones y no precisamente conceptos. Esta confusión entre conceptos y frases y oraciones impide el aprendizaje subordinado y, por lo tanto, también el supraordinado.

Por otro lado, en el mapa inicial, al no presentar ninguna línea cruzada el maestrante no desarrolla el aprendizaje proposicional combinatorio al no vincular conceptos de ramas distintas, lo que sí sucede en el mapa final donde hay cuatro líneas cruzadas, es decir, aquí es hay presencia del proposicional combinatorio al formarse las siguientes proposiciones: "representaciones del mundo a través de experiencias", "experiencias llevan a comunidades de aprendizaje", construcción conjunta llevan a comunidades de aprendizaje" e "intercambio de experiencias". Este mapa comprueba la afirmación de Aususbel (2002) de que la mayor parte del aprendizaje proposicional es subsumidor o combinatorio (p. 28).

Sin embargo, se puede afirmar que en mapa conceptual final hay un aprendizaje proposicional combinatorio y, al mismo tiempo, supraordinado en la proposición "intercambio de experiencias" porque reúne tanto dos conceptos de ramas diferentes pero el sentido que el maestrante le dio a la flecha es hacia arriba. Este caso excepcional en este mapa podría ser resuelto con una entrevista al maestrante para que dilucidara el sentido que le quiso dar a esta proposición, el matiz al significado que le quiso dar: si se trata de una relación subordinada o supraordinada.

\section{DIFICULTADES}

A pesar de los notorios avances del primer mapa al segundo, se detectan aún algunas dificultades. La primera es la pregunta de enfoque que tal como se redactó no es la más adecuada. Es una pregunta que especifique claramente el problema o la cuestión del mapa conceptual que debe construir a resolver. En este caso, la pregunta elaborada tendría que definir la naturaleza del constructivismo desde la filosofía, la psicología o la pedagogía, de lo cual no trata obviamente el mapa.

Como lo afirman Novak y Cañas, (2008) cada mapa conceptual responde a una pregunta de enfoque, y una buena pregunta de enfoque puede llevar a un mapa conceptual mucho más rico. En el aprendizaje de mapas conceptuales, los aprendices tienden a desviarse de la pregunta de enfoque y construir un mapa conceptual que puede estar relacionado con el dominio disciplinar o de la asignatura, 
pero que no responde a la pregunta. A menudo se afirma que el primer paso para aprender acerca de algo es hacer las preguntas correctas (p. 12).

Se muestra también que al final de la lectura hay menos aplicación de habilidades de análisis y de abstracción (por ejemplo, en las dos últimas ramas), pero sin ninguna intención de defender al maestrante, puede deberse a la rapidez exigida de las actividades de la MEB, no les permite leer a profundidad la totalidad del material.

Aunque se presenta con mucho menor frecuencia la confusión entre conceptos y frases y oraciones sigue apareciendo aun cuando se trabajaron los cambios de verbos en conceptos, pero es quizá uno de los aspectos donde es difícil modificar los conocimientos previos de los profesores.

El otro aspecto que muestra también aún fuertes dificultades, aunque se reconocen los avances, es la habilidad de relación. De hecho, en el segundo mapa es la que muestra más resistencia al cambio conceptual. Un poco menos del 50\% de las palabras de enlace o conectores no están bien elaboradas, lo que no impide que se logre el aprendizaje de proposiciones, pero si resta significatividad al aprendizaje. Una de las razones de la resistencia a modificar es que, en niveles de educación básica, preparatoria y en la normal, se entiende como mapa conceptual únicamente la exposición de conceptos y cuando se permiten palabras de enlace la consigna es que solo debe ser una palabra, generalmente artículos o conjunciones.

Lo antes expuesto limita el poder de aprendizaje de las proposiciones que, como ya hemos visto, es para Ausubel el mayor aprendizaje significativo que se puede desarrollar. Hay que enfatizar que por medio de las proposiciones que se elaboran en los mapas conceptuales se construyen nuevos conocimientos relacionando los nuevos conceptos al conocimiento que ya ostentamos (Cañas, 2005, p. 2). Si la redacción de proposiciones no es lógica, completa, el aprendizaje obtenido será deficiente.

En otro sentido, ambos mapas aquí analizados confirman que los mapas conceptuales tienden a ser mayormente descriptivos en lugar de explicativos, siendo muchos de ellos clasificatorios (Cañas y Novak, 2006, s. p.), como los que aquí se mostraron.

\section{CONCLUSIONES}

Como puede apreciarse en la Tabla 2, hay evidencias suficientes para evaluar las habilidades de pensamiento en la elaboración de mapas conceptuales. El mapa final, una vez explicadas y explicitadas las habilidades de pensamiento, ayudó sensiblemente al maestrante a captar el significado de los materiales que tenía que aprender (Novak y Gowin, 1998, p. 19), lo que confirma los hallazgos de otras investigaciones que ayudan a comprender los textos. 
Confirma también que no es fácil modificar los conocimientos previos acerca de la construcción de mapas conceptuales y que la única solución al problema de las concepciones erróneas y superarlas es ayudar a los estudiantes a aprender significativamente enseñándoles los mapas conceptuales tal como sugieren las ideas de Ausubel y de los colaboradores de Novak y Cañas, junto con las habilidades de pensamiento sugeridas.

Lo que hacen poderosos a los mapas conceptuales para la facilitación de aprendizajes significativos es que sirven como una especie de plantilla o andamio para ayudar a organizar y estructurar el conocimiento, a través de pequeñas unidades de interacción entre conceptos y marcos proposicionales por lo que aprendices y profesores, como es el caso del autor, se sorprenden al ver cómo esta sencilla herramienta procura el aprendizaje significativo y la creación de conjuntos de conocimiento de gran potencia que permiten la utilización del conocimiento en nuevos temas, pero también la conservación de los conocimientos durante largos periodos de tiempo (Novak y Cañas, 2008, p. 8).

Una de las virtudes para sugerir la elaboración de mapas conceptuales con el software CmapTools es que originan un tipo especial de aprendizaje: el icónico. El programa permite integrar dentro de un concepto imágenes, fotos, videos, tablas, páginas Web u otros mapas conceptuales, permitiendo una diversificación muy rica de recursos para el aprendizaje. Como lo han manifestado Novak y Cañas (2008) la integración de distintos tipos de imágenes en la mente de los alumnos crea una memoria icónica, que abona una valiosa capacidad para recordar conceptos a través de las imágenes (pp. 8-9).

La mediación pedagógica proporcionada por el profesor debe dirigirse a:

- La construcción de mapas conceptuales basados en la teoría del aprendizaje significativo y en las orientaciones de los creadores de los mapas conceptuales como Novak y sus colaboradores y no en divulgadores que obvian lo más importante de estos organizadores gráficos.

- Ayudar a los estudiantes a reconocer que todos los conceptos están en cierto modo relacionados entre sí. Por lo tanto, es necesario ser selectivo en la identificación de palabras de enlace que conectan los conceptos.

- Que los estudiantes inician a centrase en buenas y correctas palabras de enlace, se debe fomentar que realicen enlaces cruzados, ya que cada concepto puede estar relacionado con todos los demás. Se trata de enlaces entre los conceptos en distintas ramas de conocimiento en el mapa que sugieren cómo estos dominios están vinculados entre sí. Pero este proceso no debe verse como algo mecánico, sino que hay que ayudarles a identificar a los más adecuados y útiles enlaces cruzados. Son transcendentales porque demuestran que el estudiante comprende las relaciones entre las distintas ramas de conocimiento en el mapa (Novak y Cañas, 2008, p. 13). 
- La elaboración de mapas conceptuales según el modelo propuesto por Novak y colaboradores, ha demostrado al menos en este grupo de maestrantes, que son un buen medio para la formación de habilidades de pensamiento siempre y cuando haya una adecuada mediación pedagógica sobre todo basada en las propuestas del creador inicial, y sus colaboradores, de los mapas conceptuales y no en otros autores que introducen elementos que no coinciden con la teoría del aprendizaje que sustenta los mapas conceptuales.

- Que el estudiante asuma una actitud de mantenerse activo en la propia construcción de su aprendizaje, que privilegie la memorización comprensiva procure la aplicación del conocimiento.

- Orientar también a la construcción de mapas conceptuales explicativos dejando en segundo término los descriptivos y clasificatorios.

Por lo tanto, este trabajo señala una vía de actualización de los docentes de los diferentes niveles educativos a través de cursos de capacitación y actualización que se ajusten a la teoría del aprendizaje significativo de Ausubel y la construcción de mapas conceptuales en el trabajo desarrollado inicialmente por Novak y sus colaboradores.

Lo anterior adquiere mayor relevancia cuando la Secretaría de Educación Pública establece en el perfil de egreso de los estudiantes de Educación Básica que deben reflexionar sobre sus procesos de pensamiento apoyándose en organizadores gráficos para representarlos y evaluar su efectividad (SEP, 2017, p. 97). Y como lo muestra la experiencia del autor, uno de los organizadores gráficos más efectivos son los mapas conceptuales. 


\section{REFERENCIAS}

Ausubel, D. P. (1976). Psicología educativa. Un punto de vista cognoscitivo. México: Trillas.

Ausubel, D. P. (2002). Adquisición y retención del conocimiento. Una perspectiva cognitiva. Barcelona: Paidós.

Ausubel, D. P., Novak, J. D. y Hanesian, H. (1983). Psicología educativa. Un punto de vista cognoscitivo. México: Trillas

Boggino, N. (2006). Cómo elaborar mapas conceptuales. Aprendizaje significativo y globalizado. México: Homo Sapiens Ediciones.

Cañas, A. J. (diciembre-enero, 2005). Los mapas conceptuales: una ventana a la mente del estudiante. Revista Magisterio, (18), 1-6. Recuperado de http://bibliotecadigital.magisterio.co/revista/no-18-mapasconceptuales-mapas-mentales

Cañas, A. J. \& Novak, J. D. (2009). ¿Por qué la Pregunta de Enfoque? Institute for Human and Machine Cognition. Recuperado de https://cmap.ihmc.us/docs/preguntadeenfoque.php

Cañas, A. J. y Novak, J. D. (2006). Re-examinando los fundamentos para el uso efectivo de mapas conceptuales. Florida: Institute for Human and Machine Cognition. Recuperado de: http://184.182.233.150/rid=1273184479075_1424047876_41120/ReExaminando\%20los\%20Fundmento s\%20para\%20el\%20Uso\%20Efectivo\%20de\%20Mapas\%20Conceptuales.pdf

Cañas, A. J. \& Novak, J. D. (2009). ¿Qué es un mapa conceptual? Recuperado de https://cmapspublic.ihmc.us/rid=1H0LTXFDX-

1MFZVJ3YJR/Qu\%C3\%A9\%20es\%20un\%20Mapa\%20Conceptual.pdf

Dussel, E. (1985). La producción teórica de Marx. Un comentario a los Grundrisse. México: Siglo XXI Ed.

Gutiérrez, B. A. (2001). Mapas conceptuales en la enseñanza de la historia. México: Universidad de Guadalajara.

Hernández, V. (2007). Mapas conceptuales. La gestión del conocimiento en la didáctica. México: Alfaomega.

Lozano, R. (2011). “De las TIC a las TAC: tecnologías del aprendizaje y del conocimiento”. En Anuario

ThinkEPI, 5, pp. 45-47. https://recyt.fecyt.es/index.php/ThinkEPI/article/viewFile/30465/16032

Luna, V. (2014). Mapas conceptuales para favorecer el aprendizaje significativo en Ciencias de la Salud. Investigación en Educación Médica, 3(12), 220-223. Recuperado de: http://www.redalyc.org/articulo.oa?id=349733967009

Khodadady, E. \& Ghanizadeh, A. (December, 2011). The Impact of Concept Mapping on ELF Learner's Critical Thinking Ability. English Language Teaching, 4(4), 49-60. Recuperado de https://files.eric.ed.gov/fulltext/EJ1081144.pdf

Martín, E. y Solé, I. (2008). El aprendizaje significativo y la teoría de la asimilación. En César Coll, Jesús Palacios y Álvaro Marchesi (compls.). Desarrollo psicológico y educación. 2. Psicología de la educación escolar (pp. 89-116). España: Alianza Editorial. 
Maya, A. y Díaz, N. (2013). Mapas conceptuales. Su elaboración y aplicación. México: Nueva Editorial Iztaccihuatl.

Novak, J. D. (agosto, 2006). Del origen de los mapas conceptuales al desarrollo de CmapTools. En Eduteka. Tecnologías de Información y Comunicaciones para la Enseñanza Básica y Media, 1-9. Recuperado de http://www.eduteka.org/Entrevista22.php

Novak, J. D. y Cañas, A. J. (2008). La teoría subyacente a los Mapas Conceptuales y cómo construir y utilizarlos. Florida: Instituto de Cognición Humana y de la Máquina. Recuperado de https://cmap.ihmc.us/Publications/researchpapers/teoriaCmaps/teoriasubyacentemapasconceptuales-

ORIG.html

Novak, J. D. y Gowin, D. B. (1998). Aprendiendo a aprender. México: Martínez Roca.

Pérez, A. (2018). Pertinencia de la educación: caso de la maestría en gestión del desarrollo social. En E. Velázquez y O. Rahmses (coords.). Educación ambiental. Aportaciones multidisciplinarias para el desarrollo (107-128). México: Universidad Autónoma Chapingo.

Rojas, R. (2014). El proceso de investigación científica. México: Trillas.

Sánchez, J. (julio-septiembre, 2008). Una propuesta conceptual para diferenciar los programas de postgrado profesionalizantes y orientados a la investigación para la regulación, el diseño y la implementación de los programas de postgrado. Ciencia y Sociedad, 23(3), 327-341. Recuperado de http://www.redalyc.org/articulo.oa?id=87011545002

SEP (2017). Aprendizajes clave para la educación Integral. Plan y programas de estudio para la educación básica. México: Autor.

UPN (2012). Lineamientos de operación del Posgrado en Educación Básica. México: Autor. 